\title{
X-ray diffraction study of crystallite size-distribution and strain in carbon blacks
}

\author{
T. Ungár, J. Gubicza, G. Ribárik and T. W. Zerda ${ }^{1}$, \\ Department of General Physics, Eötvös University Budapest \\ H-1518, P.O.B. 32 Budapest, Hungary; \\ ${ }^{1}$ Department of Physics, Texas Christian University, Fort Worth, TX U.S.A.
}

\begin{abstract}
The crystallite size and size-distribution in the presence of strain is determined in carbon blacks by a recently developed procedure of X-ray diffraction peak profile analysis. The Fourier coefficients of the measured physical profiles are fitted by Fourier coefficients of well established $a b$ initio functions of size and strain peak profiles. Strain anisotropy is accounted for by the dislocation model of the mean square strain in terms of average dislocation contrast factors. Crystallite shape anisotropy is modelled by ellipsoids incorporated into the size profile function. The Fourier transforme of the size profile is given as an explicite formula making the fitting procedure fast. The method is applied to carbon balcks terated at different preassures and temperatures. The microstructure is characterised in terms of crystallite size-distribution, dislocation density and crystallite shape anisotropy.
\end{abstract}

\section{INTRODUCION}

X-ray diffraction peak profile analysis is a widely used method for the determination of crystallite size and lattice defect structure in crystalline materials. The evaluation procedures are based on the diffraction order dependence of size and strain. In the simplest case size is independent and strain is dependent on diffraction order. Both dependencies become, however complicated if shape or strain are anisotropic. Shape anisotropy can be modelled by cilindrical or flat bodies [1], strain anisotropy has been account for either by a phenomenological model of anisotropic elastic constants [2] or by the anisotropic strain fields of dislocations $[3,4]$. The crystallite size-distribution has recently been suggested to be determined by whole profile fitting in the absence of strain by using theoretically calculated size profiles [5]. The strain profile in dislocated crystals has been derived by Wilkens in the form of Forier transforms [6]. In the present work a procedure is described in which the Fourier coefficients of the experimentally determined physical peak profiles are fitted by the Fourier transforms of $a b$ initio size and strain profiles. The only fitting parameters are well established physical parameters characteristic of the microstructure: (i) $m$ and (ii) $\sigma$, the median and the variance of the log-normal crystallite size distribution function, (iii) $\rho$ and (iv) $M$, the density and the arrangement parameter of dislocations and (v) $q$ or $A$ and $B$, the relevant parameters describing the average dislocation contrast factors in cubic or hexagonal crystals, respectively. In the case of shape anisotropy a further fitting parameter can be introduced to characterise, for example, the ellipticity of crystallite shape. The procedure will be illustrated by the application to representative carbon black specimens. A more detailed account of the results is given and discussed in an other paper of the present proceedings [7]. 


\section{EVALUATION OF X-RAY DIFFRACTION PROFILES}

Within the kinematical theory of X-ray diffraction the physical profile of a Bragg reflection is given by the convolution of the size and the distortion profiles [8]:

$$
\mathrm{I}^{\mathrm{F}}=\mathrm{I}^{\mathrm{S}} * \mathrm{I}^{\mathrm{D}}
$$

where the superscripts S and D stand for size and distortion, respectively. The Fourier transform of this equation is known as the Warren-Averbach method [8]:

$$
\ln A(L) \cong \ln A_{L}^{S}-2 \pi^{2} L^{2} g^{2}<\varepsilon_{g, L}^{2}>,
$$

where $A(L)$ are the absolute values of the Fourier coefficients of the physical profiles, $A_{L}{ }^{S}$ are the size Fourier coefficients, $g$ is the absolute value of the diffraction vector and $\left\langle\varepsilon_{g, L}{ }^{2}\right\rangle$ is the mean square strain. $L$ is the Fourier length defined as $L=n a_{3}$ [8], where $a_{3}=\lambda / 2\left(\sin \theta_{2}-\sin \theta_{1}\right), \mathrm{n}$ are integers starting from zero, $\lambda$ is the wavelength of X-rays and $\left(\theta_{2}-\theta_{1}\right)$ is the angular range of the measured diffraction profile. Equations (1) and (2) tell that the size and strain profiles are in convolution and the Fourier coefficients are in product. Once we know these functions it is only a question of skillful numerical calculus to make a fitting between experiment and theory. The Fourier transform of the size profile for ellipsoidal crystallite shape with log-normal size distribution can be given as [9]:

$$
\begin{aligned}
& A^{\mathrm{S}}(\mathrm{L}) \sim \frac{m_{h k l}{ }^{3} \exp \left(4.5 \sigma^{2}\right)}{3} \operatorname{erfc}\left[\frac{\ln \left(|L| / m_{h k l}\right)}{\sqrt{2} \sigma}-1.5 \sqrt{2} \sigma\right]- \\
& -\frac{m_{h k l}{ }^{2} \exp \left(2 \sigma^{2}\right)|L|}{2} \operatorname{erfc}\left[\frac{\ln \left(|L| / m_{h k l}\right)}{\sqrt{2} \sigma}-\sqrt{2} \sigma\right]+\frac{||^{3}}{6} \operatorname{erfc}\left[\frac{\ln \left(|L| / m_{h k l}\right)}{\sqrt{2} \sigma}\right] .
\end{aligned}
$$

where $\operatorname{erfc}$ is the complementary error function, $\sigma$ is the variance and $m_{h k l}$ is the median of the log-normal size distribution function in the $h k l$ direction. $m_{h k l}$ depends on the indeces of reflections in the following way:

$$
m_{h k l}=\frac{m}{1+\left(\frac{1}{\varepsilon^{2}}-1\right)\left(\frac{l^{2}}{\frac{4}{3} \frac{c^{2}}{a^{2}}\left(h^{2}+k^{2}+h k\right)+l^{2}}\right)},
$$

where $m$ is the median of the size distribution paralel to the hexagonal basal plane, $\mathrm{c} / \mathrm{a}$ is the ratio of the lattice constants of the hexagonal crystal and $\varepsilon$ is the aspect ratio of the ellipsoid. For spherical crystallites with log-normal size distribution the area-, volume- and arithmetically weighted mean crystallite sizes are obtained as [10]: 


$$
\begin{aligned}
& <\mathrm{x}>_{\text {area }}=\mathrm{m} \exp \left(2.5 \sigma^{2}\right) . \\
& <\mathrm{x}>_{\mathrm{vol}}=\mathrm{m} \exp \left(3.5 \sigma^{2}\right) . \\
& <\mathrm{x}>_{\text {arithm }}=\mathrm{m} \exp \left(0.5 \sigma^{2}\right) .
\end{aligned}
$$

The mean square strain in dislocated crystals [6]:

$$
<\varepsilon_{g, L}^{2}>=(b / 2 \pi)^{2} \pi \rho C f(\eta),
$$

where $\eta \sim L / R_{\mathrm{e}}, R_{\mathrm{e}}$ is the effective outer cut off radius of dislocations, $b, \rho$ and $C$ are the Burgers vector, the density and the contrast factors of dislocations and $f(\eta)$ is the $L$ dependence of the mean square strain in a dislocated crystal. In the following $f(\eta)$ will be called the Wilkens function. This function starts with a logarithmic term and continue as a hyperbola for large $L$ values:

$$
\begin{array}{ll}
f(\eta) \sim-\ln \eta+\left(\frac{7}{4}-\ln 2\right)+\ldots & \text { for } \eta \leq 1 \\
f(\eta) \sim-\frac{512}{90 \pi} \frac{1}{\eta}+\ldots & \text { for } \eta \geq 1
\end{array}
$$

The detailed expressions for $f(\eta)$ are given in equations A.6 to A.8 in [6] and have been cited in eqs. (22) and (23) in [9]. In the procedure developed here the full expressions in A.6 to A.8 in [6] were used.

The average dislocation contrast factors are the weighted average of the individual $C$ factors either over the dislocation population or over the permutations of the $h k l$ indices $[11,12]$. Based on the theory of line broadening caused by dislocations it can been shown that in an untextured cubic and hexagonal polycrystalline specimen the values of $\bar{C}$ are simple functions of the invariants of the fourth order polynomials of $h k l[13]$ :

$$
\bar{C}=\bar{C}_{h 00}\left(1-q H^{2}\right)
$$

and

$$
\bar{C}=\bar{C}_{h k 0}\left[1+\frac{\left[A\left(h^{2}+k^{2}+(h+k)^{2}\right)+B l^{2}\right] l^{2}}{\left[h^{2}+k^{2}+(h+k)^{2}+\frac{3}{2}\left(\frac{a}{c}\right)^{2} l^{2}\right]^{2}}\right],
$$

respectively, where $\bar{C}_{h 00}$ and $\bar{C}_{h k 0}$ are the average dislocation contrast factors for the $h 00$ and $h k 0$ reflections, respectively, $H^{2}=\left(h^{2} k^{2}+h^{2} l^{2}+k^{2} l^{2}\right) /\left(h^{2}+k^{2}+l^{2}\right)^{2} ; q, A$ and $B$ are parameters depending on the elastic constants and on the character of dislocations (e.g. edge or screw type) in the crystal and c/a is the ratio of the two lattice constants of the hexagonal crystal. 


\section{CORRECTIONS FOR INSTRUMENTAL EFFECTS, BACKGROUND AND OVERLAPPING PEAKS}

In the present case strong overlap of the diffraction profiles has been observed. The overlapping peaks have to be separated since the present evaluation method is worked out for individual profiles. Background subtraction and the separation of overlapping peaks is carried out in one step. Two or more analytical functions, usually of PearsonVII or Pseudo-Voigt type plus a linear background are fitted to the overlapping peaks. In the next step the unwanted fitted peaks together with the linear background are subtracted leaving the targeted peak free of overlap and background. The procedure is then repeated for the other targeted peaks. The separated profiles are taken as individual diffraction profiles in the evaluation procedures.

Due to very large strains and nanometric crystallite size peak broadening of the carbon black specimens is about two orders of magnitude larger than the instrumental effects. For this reason no instrumental corrections were necessary to be carried out. If, however, the instrumental effect can not be neglected the function fitted to the Fourier transform of the observed profiles would be the product of the size, the strain and the instrumental Fourier coefficients in accordance with Stokes correction [14].

\section{RESULTS AND DISCUSSION}

The diffraction profiles were measured in a Philips X'pert diffractometer using $\mathrm{Cu}$ anode and pyrolitic graphite secondary monochromator. In order to have good counting statistics matched with the peak brodening the step size and countimg time in one step were selected as: 0.01 Deg. $(2 \theta)$ and $10 \mathrm{sec}$, respectively. Two typical powder patterns are shown in Fig. 1.

\section{ACKNOWLEDGEMENTS}

The authors are grateful for the financial support of the Hungarian Scientific Research Fund, OTKA, Grant Nos. T031786, T029701 and D-29339.

\section{REFERENCES}

1. J. I. Langford,

2. $\quad$ P. W. Stephens, J. Appl. Cryst. 32, 281 (1999).

3. T. Ungár and A. Borbély, Appl. Phys. Lett. 69, 3173 (1996).

4. T. Ungár, I. Dragomir, Á. Révész and A. Borbély, J. Appl. Cryst. 32, 992 (1999).

5. J. I. Langford, D. Louër and P. Scardi, J. Appl. Cryst. 33, 964 (2000).

6. M. Wilkens, Fundamental Aspects of Dislocation Theory, ed. J. A. Simmons, R. deWit, R. Bullough, Vol. II. Nat. Bur. Stand. (US) Spec. Publ. No. 317, Washington, DC. USA, p. 1195(1970).

7. T. W. Zerda and T. Ungár, Proc. MRS Fall meeting, Symp: Filled and Nanocomposite Polymer Materials, eds. R. P. Hjelm, A. I. Nakatani, M. Gerspacher, R. Krishnamoorti, Boston, U.S.A. (2000).

8. B. E. Warren, Progr. Metal Phys. 8, 147 (1959).

9. G. Ribárik, T. Ungár and J. Gubicza, J. Appl. Cryst. submitted for publication.

10. W. C. Hinds, Aerosol Technology: Properties, Behavior and Measurement of 
Airborne Particles, Wiley, New York, (1982).

11. M. A. Krivoglaz, in Theory of X-ray and Thermal Neutron Scattering by real Crystals, Plenum Press, N. Y. 1969; and in X-ray and Neutron Diffraction in Nonideal Crystals, Springer-Verlag, Berlin Heidelberg New York, (1996).

12. P. Klimanek and R. Kuzel Jr., J. Appl. Cryst. 21, 59, 363 (1988); 22, 299 (1989).

13. T. Ungár and G. Tichy, phys. stat. sol. (a) 171, 425 (1999).

14. A. R. Stokes, Proc. Phys. Soc. 61, 382 (1948). 